\title{
Lazer-Leach Type Conditions on Periodic Solutions of Liénard Equation with a Deviating Argument at Resonance
}

\author{
Zaihong Wang \\ School of Mathematical Sciences, Capital Normal University, Beijing 100048, China \\ Correspondence should be addressed to Zaihong Wang; zhwang@cnu.edu.cn \\ Received 28 February 2013; Accepted 15 April 2013 \\ Academic Editor: Wing-Sum Cheung
}

Copyright ( 2013 Zaihong Wang. This is an open access article distributed under the Creative Commons Attribution License, which permits unrestricted use, distribution, and reproduction in any medium, provided the original work is properly cited.

We study the existence of periodic solutions of Liénard equation with a deviating argument $x^{\prime \prime}+f(x) x^{\prime}+n^{2} x+g(x(t-\tau))=$ $p(t)$, where $f, g, p: R \rightarrow R$ are continuous and $p$ is $2 \pi$-periodic, $0 \leq \tau<2 \pi$ is a constant, and $n$ is a positive integer. Assume that the $\operatorname{limits}_{\lim _{x \rightarrow \pm \infty}} g(x)=g( \pm \infty)$ and $\lim _{x \rightarrow \pm \infty} F(x)=F( \pm \infty)$ exist and are finite, where $F(x)=\int_{0}^{x} f(u) d u$. We prove that the given equation has at least one $2 \pi$-periodic solution provided that one of the following conditions holds: $2 \cos (n \tau)[g(+\infty)-g(-\infty)] \neq \int_{0}^{2 \pi} p(t) \sin (\theta+n t) d t$, for all $\theta \in[0,2 \pi], 2 n \cos (n \tau)[F(+\infty)-F(-\infty)] \neq \int_{0}^{2 \pi} p(t) \sin (\theta+n t) d t$, for all $\theta \in$ $[0,2 \pi], 2[g(+\infty)-g(-\infty)]-2 n \sin (n \tau)[F(+\infty)-F(-\infty)] \neq \int_{0}^{2 \pi} p(t) \sin (\theta+n t) d t$, for all $\theta \in[0,2 \pi], 2 n[F(+\infty)-F(-\infty)]-$ $2 \sin (n \tau)[g(+\infty)-g(-\infty)] \neq \int_{0}^{2 \pi} p(t) \sin (\theta+n t) d t$, for all $\theta \in[0,2 \pi]$.

\section{Introduction}

We are concerned with the existence of periodic solutions of Liénard equation with a deviating argument as follows:

$$
x^{\prime \prime}+f(x) x^{\prime}+n^{2} x+g(x(t-\tau))=p(t),
$$

where $f, g, p: \mathbf{R} \rightarrow \mathbf{R}$ are continuous and $p$ is $2 \pi$-periodic, $0 \leq \tau<2 \pi$ is a constant, and $n$ is a positive integer.

In recent years, the periodic problem of Liénard equations with a deviating argument has been widely studied because of its background in applied sciences (see [1-8] and the references cited therein).

In the case when $f(x) \equiv 0$, for all $x \in \mathbf{R}$ and $\tau=0$, (1) becomes

$$
x^{\prime \prime}+n^{2} x+g(x)=p(t) .
$$

Assume that limits

$$
\left(h_{1}\right) \lim _{x \rightarrow \pm \infty} g(x)=g( \pm \infty)
$$

exist and are finite. Lazer and Leach [9] proved that (2) has at least one $2 \pi$-periodic solution provided that the following condition holds:

$$
\begin{aligned}
& 2[g(+\infty)-g(-\infty)] \\
& \quad \neq \int_{0}^{2 \pi} p(t) \sin (\theta+n t) d t, \quad \forall \theta \in[0,2 \pi] .
\end{aligned}
$$

Assume that, besides $\left(h_{1}\right)$, the limits

$$
\left(h_{2}\right) \lim _{x \rightarrow \pm \infty} F(x)=F( \pm \infty)
$$

exist and are finite, where $F(x)=\int_{0}^{x} f(u) d u$. It was proved in $[10]$ that the following equation:

$$
x^{\prime \prime}+f(x) x^{\prime}+n^{2} x+g(x(t-\tau))=p(t)
$$


has at least $2 \pi$-periodic solution provided that one of the following conditions holds:

$$
\begin{aligned}
& 2[g(+\infty)-g(-\infty)] \\
& \quad \neq \int_{0}^{2 \pi} p(t) \sin (\theta+n t) d t, \quad \forall \theta \in[0,2 \pi], \\
& 2 n[F(+\infty)-F(-\infty)] \\
& \quad \neq \int_{0}^{2 \pi} p(t) \sin (\theta+n t) d t, \quad \forall \theta \in[0,2 \pi] .
\end{aligned}
$$

In the case when $f(x) \equiv 0$, for all $x \in \mathbf{R}$ and $\tau \neq 0$, (1) becomes as follows:

$$
x^{\prime \prime}+n^{2} x+g(x(t-\tau))=p(t)
$$

When the condition $\left(h_{1}\right)$ holds, it was proved in [5] that (6) has at least one $2 \pi$-periodic solution provided that the condition (3) holds.

In the present paper, we deal with the existence of periodic solutions of (1) by assuming $\left(h_{1}\right)$ and $\left(h_{2}\right)$. By using the continuation theorem [11], we prove the following result.

Theorem 1. Assume that the conditions $\left(h_{1}\right)$ and $\left(h_{2}\right)$ hold. Then (1) has at least one $2 \pi$-periodic solution provided that one of the following conditions is satisfied:

$$
\begin{aligned}
& 2 \cos (n \tau)[g(+\infty)-g(-\infty)] \\
& \quad \neq \int_{0}^{2 \pi} p(t) \sin (\theta+n t) d t, \quad \forall \theta \in[0,2 \pi], \\
& 2 n \cos (n \tau)[F(+\infty)-F(-\infty)] \\
& \quad \neq \int_{0}^{2 \pi} p(t) \sin (\theta+n t) d t, \quad \forall \theta \in[0,2 \pi], \\
& 2[g(+\infty)-g(-\infty)]-2 n \sin (n \tau)[F(+\infty)-F(-\infty)] \\
& \quad \neq \int_{0}^{2 \pi} p(t) \sin (\theta+n t) d t, \quad \forall \theta \in[0,2 \pi], \\
& 2 n[F(+\infty)-F(-\infty)]-2 \sin (n \tau)[g(+\infty)-g(-\infty)] \\
& \quad \neq \int_{0}^{2 \pi} p(t) \sin (\theta+n t) d t, \quad \forall \theta \in[0,2 \pi] .
\end{aligned}
$$

Remark 2. Let us denote by $\Phi$ the function on the right-hand side of four inequalities above, namely,

$$
\Phi(\theta)=\int_{0}^{2 \pi} p(t) \sin (\theta+n t) d t, \quad \theta \in[0,2 \pi] .
$$

Then $\Phi$ can be expressed in the following form:

$$
\Phi(\theta)=A \sin \theta+B \cos \theta, \quad \theta \in[0,2 \pi],
$$

where

$$
A=\int_{0}^{2 \pi} p(t) \cos n t d t, \quad B=\int_{0}^{2 \pi} p(t) \sin n t d t .
$$

Obviously, the value of $\Phi$ forms a closed interval $[-\varrho, \varrho]$ with $\varrho=\sqrt{A^{2}+B^{2}}$. Therefore, the four conditions in Theorem 1 are equivalent to the following conditions, respectively:

$$
\begin{gathered}
2 \cos (n \tau)[g(+\infty)-g(-\infty)] \notin[-\varrho, \varrho], \\
2 n \cos (n \tau)[F(+\infty)-F(-\infty)] \notin[-\varrho, \varrho], \\
2[g(+\infty)-g(-\infty)] \\
\quad-2 n \sin (n \tau)[F(+\infty)-F(-\infty)] \notin[-\varrho, \varrho], \\
2 n[F(+\infty)-F(-\infty)] \\
\quad-2 \sin (n \tau)[g(+\infty)-g(-\infty)] \notin[-\varrho, \varrho] .
\end{gathered}
$$

Remark 3. In the case when $\tau=0$, the four conditions in Theorem 1 reduce to the conditions (5). Therefore, Theorem 1 generalizes the result in [10].

Throughout this paper, we always use $\mathbf{R}$ to denote the real number set. For a multivariate function $\zeta$ depending on $r$, the notation $\zeta=o(1)$ always means that, for $r \rightarrow \infty, \zeta \rightarrow 0$ holds uniformly with respect to other variables, whereas $\zeta=$ $O(1)$ (or $\zeta=O\left(r^{-1}\right)$ ) always means that $\zeta$ (or $r \cdot \zeta$ ) is bounded for $r$ large enough. For any continuous $2 \pi$-periodic function $\psi(t)$, we always set $\|\psi\|_{\infty}=\max _{0 \leq t \leq 2 \pi}|\psi(t)|$.

\section{Basic Lemmas}

It is well known that continuation theorems play an important role in studying the existence of periodic solutions of differential equations. We now introduce a continuation theorem which will be used to prove the existence of periodic solutions of (1).

Let $X$ and $Y$ be two real Banach spaces and let $L: D(L) \subset$ $X \rightarrow Y$ be a Fredholm operator with index zero, where $D(L)$ denotes the domain of $L$. This means that $\operatorname{Im} L$ is a closed subspace of $Y$ and $\operatorname{dimker} L=\operatorname{codim} \operatorname{Im} L<+\infty$. Let $P: X \rightarrow \operatorname{Ker} L, Q: Y \rightarrow Y$ be two linear continuous projectors satisfying the following:

$$
\operatorname{Im} P=\operatorname{Ker} L, \quad \operatorname{Ker} Q=\operatorname{Im} L .
$$

Then we have the following:

$$
X=\operatorname{Ker} L \oplus \operatorname{Ker} P, \quad Y=\operatorname{Im} L \oplus \operatorname{Im} Q .
$$

Clearly, $L_{P}=\left.L\right|_{D(L) \cap \operatorname{Ker} P} \rightarrow \operatorname{Im} L$ is invertible. Denote by $K_{P}$ the inverse of $L_{P}$. Let $\Omega \subset X$ be an open bounded set. A continuous map $N: \bar{\Omega} \rightarrow Y$ is said to be $L$-compact on $\bar{\Omega}$ if both $Q N: \bar{\Omega} \rightarrow Y$ and $K_{P}(I-Q) N: \bar{\Omega} \rightarrow X$ are compact.

Lemma 4 (see [11]). Let $X$ and $Y$ be two real Banach spaces. Suppose that $L: D(L) \subset X \rightarrow Y$ is a Fredholm operator with index zero and $N: \bar{\Omega} \rightarrow Y$ is L-compact on $\bar{\Omega}$, where $\Omega$ is an open bounded subset of $X$. Moreover, assume that all the following conditions are satisfied:

(1) $L x \neq \lambda N x$, for all $x \in \partial \Omega \cap D(L), \lambda \in(0,1)$;

(2) $N x \notin \operatorname{Im} L$, for all $x \in \partial \Omega \cap \operatorname{Ker} L$; 
(3) The Brouwer degree $\operatorname{deg}\{J Q N, \Omega \cap \operatorname{Ker} L, 0\} \neq 0$, where $J: \operatorname{Im} Q \rightarrow \operatorname{Ker} L$ is an isomorphism.

Then equation $L x=N x$ has at least one solution on $D(L) \cap \bar{\Omega}$.

\section{Main Results}

In this section, we will use the continuation theorem introduced in Section 2 to prove the existence of periodic solutions of (1). To this end, we first quote some notations and definitions.

Let $X$ and $Y$ be two Banach spaces defined by the following:

$$
\begin{gathered}
X=\left\{x \in C^{1}(\mathbf{R}, \mathbf{R}): x(t+2 \pi)=x(t), \forall t \in \mathbf{R}\right\}, \\
Y=\{y \in C(\mathbf{R}, \mathbf{R}): y(t+2 \pi)=y(t), \forall t \in \mathbf{R}\}
\end{gathered}
$$

with the following norms

$$
\|x\|_{X}=\max \left\{\|x\|_{\infty},\left\|x^{\prime}\right\|_{\infty}\right\}, \quad\|y\|_{Y}=\|y\|_{\infty} .
$$

Define a linear operator

$$
L: D(L) \subset X \longrightarrow Y, \quad L x=x^{\prime \prime}+n^{2} x
$$

where $D(L)=\left\{x \in X: x^{\prime \prime} \in C(\mathbf{R}, \mathbf{R})\right\}$, and a nonlinear operator

$$
\begin{gathered}
N: \mathrm{X} \longrightarrow Y, \\
(N x)(t)=-f(x(t)) x^{\prime}(t)-g(x(t-\tau))+p(t) .
\end{gathered}
$$

It is easy to see that

$$
\operatorname{Ker} L=\operatorname{Span}\{\sin n t, \cos n t\},
$$

$$
\begin{gathered}
\operatorname{Im} L=\left\{y \in Y: \int_{0}^{2 \pi} y(t) \sin n t d t=0,\right. \\
\left.\int_{0}^{2 \pi} y(t) \cos n t d t=0\right\} .
\end{gathered}
$$

On the other hand, for any $y \in Y$, we can write the following:

$$
y(t)=\bar{y}(t)+\tilde{y}(t), \quad \forall t \in[0,2 \pi],
$$

where $\bar{y}(t)$ is defined by the following:

$$
\bar{y}(t)=a \sin n t+b \cos n t
$$

with

$$
a=\frac{1}{\pi} \int_{0}^{2 \pi} y(t) \sin n t d t, \quad b=\frac{1}{\pi} \int_{0}^{2 \pi} y(t) \cos n t d t,
$$

whereas $\tilde{y}(t)$ satisfies the following:

$$
\int_{0}^{2 \pi} \tilde{y}(t) \sin n t d t=0, \quad \int_{0}^{2 \pi} \tilde{y}(t) \cos n t d t=0 .
$$

Therefore,

$$
Y=\operatorname{Ker} L \oplus \operatorname{Im} L \text {. }
$$

It follows that $L$ is a Fredholm map of index zero.

Let us define two continuous projectors $P: X \rightarrow \operatorname{Ker} L$ and $Q: Y \rightarrow Y$ by setting the following:

$$
\begin{gathered}
(P x)(t)=a_{1} \sin n t+b_{1} \cos n t \\
(Q y)(t)=\bar{y}(t)=a \sin n t+b \cos n t, \quad \forall t \in[0,2 \pi]
\end{gathered}
$$

for any $x \in X$ and $y \in Y$, where constants $a_{1}$ and $b_{1}$ are defined as constants $a$ and $b$. Obviously, $\operatorname{Im} Q=\operatorname{Ker} L$.

Set $L_{P}=\left.L\right|_{D(L) \cap \operatorname{Ker} P} \rightarrow \operatorname{Im} L$. Then $L_{P}$ is an algebraic isomorphism and we define $K_{P}: \operatorname{Im} L \rightarrow D(L) \cap \operatorname{Ker} P$ by the following:

$$
K_{P}=L_{P}^{-1}
$$

Clearly, we have that, for any $y \in \operatorname{Im} L$,

$$
\begin{aligned}
\left(K_{P} y\right)(t)= & \frac{1}{n} \int_{0}^{t} y(s) \sin n(t-s) d s \\
& +\frac{1}{2 n \pi} \int_{0}^{2 \pi} s y(s) \sin n(t-s) d s .
\end{aligned}
$$

For any open bounded set $\Omega \subset X$, we can prove by standard arguments that $K_{P}(I-Q) N$ and $Q N$ are compact on the closure $\bar{\Omega}$. Therefore, $N$ is $L$-compact on $\bar{\Omega}$.

It is noted that (1) is equivalent to the operator equation

$$
L x=N x .
$$

To use Lemma 4, we embed this operator equation into an equation family with a parameter $\lambda \in(0,1)$,

$$
L x=\lambda N x,
$$

which is equivalent to the equation as follows:

$$
\begin{aligned}
x^{\prime \prime}+ & n^{2} x+\lambda f(x(t)) x^{\prime}(t)+\lambda g(x(t-\tau)) \\
& =\lambda p(t), \quad \lambda \in(0,1) .
\end{aligned}
$$

In the following, we will prove some new results on the existence of periodic solutions of (1) by using the continuation theorem. Consider the equivalent system of (29):

$$
x^{\prime}=y-\lambda F(x), \quad y^{\prime}=-n^{2} x-\lambda g(x(t-\tau))+\lambda p(t) .
$$

Let $x(t)$ be any (possible) $2 \pi$-periodic solution of (29). Set $y(t)=x^{\prime}(t)+\lambda F(x(t))$. Then $(x(t), y(t))$ is a $2 \pi$-periodic solution of (30).

Now, let us introduce a transformation $\Phi:(r, \theta) \in \mathbf{R}^{+} \times$ $S^{1} \rightarrow(x, y) \in \mathbf{R}^{2} \backslash\{0\}$ with $S^{1}=\mathbf{R} / 2 \pi \mathbf{Z}$,

$$
x=\frac{1}{n} r^{1 / 2} \sin \theta, \quad y=r^{1 / 2} \cos \theta .
$$


Under the transformation $\Phi$, if $|x(t)|+|y(t)| \neq 0$, for $t \in$ $[0,2 \pi]$, then the $2 \pi$-periodic solution $(x(t), y(t))$ of $(30)$ can be expressed in the form $(r(t), \theta(t))$ satisfying the equations as follows:

$$
\begin{aligned}
\frac{d \theta}{d t}= & +\lambda r^{-1 / 2} g\left(\frac{1}{n} r^{1 / 2}(t-\tau) \sin \theta(t-\tau)\right) \sin \theta \\
& -\lambda n r^{-1 / 2} F\left(\frac{1}{n} r^{1 / 2} \sin \theta\right) \cos \theta-\lambda r^{-1 / 2} p(t) \sin \theta, \\
\frac{d r}{d t}= & -2 \lambda r^{1 / 2} g\left(\frac{1}{n} r^{1 / 2}(t-\tau) \sin \theta(t-\tau)\right) \cos \theta \\
& -2 \lambda n r^{1 / 2} F\left(\frac{1}{n} r^{1 / 2} \sin \theta\right) \sin \theta+2 \lambda r^{1 / 2} p(t) \cos \theta .
\end{aligned}
$$

Let us set $\left(r_{0}, \theta_{0}\right)=(r(0), \theta(0))$ with $r_{0}=n^{2} x^{2}(0)+y^{2}(0)$. Without loss of generality, we always assume $\theta(0) \in[0,2 \pi]$. Dividing the second equation of (32) by $r^{1 / 2}$, we get the following:

$$
\begin{aligned}
\frac{d r^{1 / 2}}{d t}= & -\lambda g\left(\frac{1}{n} r^{1 / 2}(t-\tau) \sin \theta(t-\tau)\right) \cos \theta \\
& -\lambda n F\left(\frac{1}{n} r^{1 / 2} \sin \theta\right) \sin \theta+\lambda p(t) \cos \theta
\end{aligned}
$$

Integrating (33) and applying conditions $\left(h_{1}\right)$ and $\left(h_{2}\right)$, we get the following:

$$
r(t)^{1 / 2}=r_{0}^{1 / 2}+O(1), \quad \forall t \in[0,2 \pi] .
$$

Furthermore,

$$
r(t)^{-1 / 2}=r_{0}^{-1 / 2}+O\left(r_{0}^{-1}\right), \quad t \in[0,2 \pi] .
$$

On the other hand, it follows from the first equation of (32) and (35) that

$$
\frac{d \theta}{d t}=n+O\left(r_{0}^{-1 / 2}\right), \quad t \in[0,2 \pi]
$$

Therefore, we get the following:

$$
\theta(t)=\theta_{0}+n t+O\left(r_{0}^{-1 / 2}\right), \quad t \in[0,2 \pi] .
$$

The estimations (34)-(37) will be used to obtain apriori bounds of $2 \pi$-periodic solutions of (29). Multiplying both sides of (29) by $\sin \left(\theta_{0}+n t\right)$ and $\cos \left(\theta_{0}+n t\right)$, respectively, and integrating over the interval $[0,2 \pi]$, we obtain the following:

$$
\begin{gathered}
\int_{0}^{2 \pi} f(x(t)) x^{\prime}(t) \sin \left(\theta_{0}+n t\right) d t \\
\quad+\int_{0}^{2 \pi} g(x(t-\tau)) \sin \left(\theta_{0}+n t\right) d t \\
=\int_{0}^{2 \pi} p(t) \sin \left(\theta_{0}+n t\right) d t \\
\int_{0}^{2 \pi} f(x(t)) x^{\prime}(t) \cos \left(\theta_{0}+n t\right) d t \\
\quad+\int_{0}^{2 \pi} g(x(t-\tau)) \cos \left(\theta_{0}+n t\right) d t \\
=\int_{0}^{2 \pi} p(t) \cos \left(\theta_{0}+n t\right) d t .
\end{gathered}
$$

Hence,

$$
\begin{aligned}
& -n \int_{0}^{2 \pi} F(x(t)) \cos \left(\theta_{0}+n t\right) d t \\
& \quad+\int_{0}^{2 \pi} g(x(t-\tau)) \sin \left(\theta_{0}+n t\right) d t \\
& =\int_{0}^{2 \pi} p(t) \sin \left(\theta_{0}+n t\right) d t \\
& n \int_{0}^{2 \pi} F(x(t)) \sin \left(\theta_{0}+n t\right) d t \\
& \quad+\int_{0}^{2 \pi} g(x(t-\tau)) \cos \left(\theta_{0}+n t\right) d t \\
& =\int_{0}^{2 \pi} p(t) \cos \left(\theta_{0}+n t\right) d t .
\end{aligned}
$$

Multiplying both sides of (29) by $\sin \left(\theta_{0}+n(t-\tau)\right)$ and $\cos \left(\theta_{0}+\right.$ $n(t-\tau))$, respectively, and integrating over the interval $[0,2 \pi]$, we obtain the following:

$$
\begin{aligned}
\int_{0}^{2 \pi} f(x(t)) x^{\prime}(t) \sin \left(\theta_{0}+n(t-\tau)\right) d t \\
\quad+\int_{0}^{2 \pi} g(x(t-\tau)) \sin \left(\theta_{0}+n(t-\tau)\right) d t \\
=\int_{0}^{2 \pi} p(t) \sin \left(\theta_{0}+n(t-\tau)\right) d t \\
\int_{0}^{2 \pi} f(x(t)) x^{\prime}(t) \cos \left(\theta_{0}+n(t-\tau)\right) d t \\
\quad+\int_{0}^{2 \pi} g(x(t-\tau)) \cos \left(\theta_{0}+n(t-\tau)\right) d t \\
=\int_{0}^{2 \pi} p(t) \cos \left(\theta_{0}+n(t-\tau)\right) d t
\end{aligned}
$$


Hence,

$$
\begin{aligned}
& -n \int_{0}^{2 \pi} F(x(t)) \cos \left(\theta_{0}+n(t-\tau)\right) d t \\
& \quad+\int_{0}^{2 \pi} g(x(t-\tau)) \sin \left(\theta_{0}+n(t-\tau)\right) d t \\
& \quad=\int_{0}^{2 \pi} p(t) \sin \left(\theta_{0}+n(t-\tau)\right) d t, \\
& n \int_{0}^{2 \pi} F(x(t)) \sin \left(\theta_{0}+n(t-\tau)\right) d t \\
& \quad+\int_{0}^{2 \pi} g(x(t-\tau)) \cos \left(\theta_{0}+n(t-\tau)\right) d t \\
& =\int_{0}^{2 \pi} p(t) \cos \left(\theta_{0}+n(t-\tau)\right) d t .
\end{aligned}
$$

Proof of Theorem 1. We shall prove the existence of periodic solutions of (1) provided that either

$$
\begin{aligned}
& 2 \cos n \tau[g(+\infty)-g(-\infty)] \\
& \quad \neq \int_{0}^{2 \pi} p(t) \sin (\theta+n t) d t, \quad \forall \theta \in[0,2 \pi],
\end{aligned}
$$

or

$$
\begin{gathered}
2 n[F(+\infty)-F(-\infty)]-2 \sin n \tau[g(+\infty)-g(-\infty)] \\
\quad \neq \int_{0}^{2 \pi} p(t) \sin (\theta+n t) d t, \quad \forall \theta \in[0,2 \pi]
\end{gathered}
$$

holds by using (39) and (40). The other cases can be handled similarly by using (43) and (44). We proceed in three steps.

(1) We prove that there exist positive constants $M_{1}$ and $M_{2}$ such that, for any $2 \pi$-periodic solution $x(t)$ of (29),

$$
\|x\|_{\infty}<M_{1}, \quad\left\|x^{\prime}\right\|_{\infty}<M_{2} .
$$

Assume by contradiction that (45) does not hold. Then there exists a sequence of $2 \pi$-periodic solutions $\left\{x_{k}(t)\right\}_{k=1}^{\infty}$ of (29) with $\lambda=\lambda_{k} \in(0,1)$ such that

$$
\left\|x_{k}\right\|_{\infty}+\left\|x_{k}^{\prime}\right\|_{\infty} \longrightarrow+\infty, \quad \text { for } k \rightarrow \infty
$$

Write $y_{k}(t)=x_{k}^{\prime}(t)+\lambda_{k} F\left(x_{k}(t)\right)$. Since $F$ is bounded on the interval $(-\infty,+\infty)$, we have the following:

$$
\left\|x_{k}\right\|_{\infty}+\left\|y_{k}\right\|_{\infty} \longrightarrow+\infty, \quad \text { for } k \rightarrow \infty .
$$

Let $\left(r_{k}(t), \theta_{k}(t)\right)$ be the $2 \pi$-periodic solution of (32) related to $\left(x_{k}(t), y_{k}(t)\right)$. Obviously, $r_{k}(t)=n^{2} x_{k}^{2}(t)+y_{k}^{2}(t)$. Then we have the following:

$$
\left\|r_{k}\right\|_{\infty} \longrightarrow+\infty, \quad \text { for } k \longrightarrow \infty \text {. }
$$

Without loss of generality, we also assume $\theta_{k}(0) \in[0,2 \pi]$. It follows from (39) that

$$
\begin{aligned}
-n \int_{0}^{2 \pi} F\left(x_{k}(t)\right) \cos \left(\theta_{k}(0)+n t\right) d t \\
\quad+\int_{0}^{2 \pi} g\left(x_{k}(t-\tau)\right) \sin \left(\theta_{k}(0)+n t\right) d t \\
=\int_{0}^{2 \pi} p(t) \sin \left(\theta_{k}(0)+n t\right) d t .
\end{aligned}
$$

From (34) and (37) we get that, for $t \in[0,2 \pi]$,

$$
\begin{aligned}
g\left(x_{k}(t-\tau)\right) & \\
& =g\left(\frac{1}{n} r_{k}(t-\tau)^{1 / 2} \sin \theta_{k}(t-\tau)\right) \\
& =g\left(\frac{1}{n} r_{k}(0)^{1 / 2} \sin \left(\theta_{k}(0)+n(t-\tau)\right)+O(1)\right) .
\end{aligned}
$$

Therefore,

$$
\begin{aligned}
\int_{0}^{2 \pi} g\left(x_{k}(t-\tau)\right) \sin \left(\theta_{k}(0)+n t\right) d t \\
=\int_{0}^{2 \pi} g\left(\frac{1}{n} r_{k}(0)^{1 / 2} \sin \left(\theta_{k}(0)+n(t-\tau)\right)+O(1)\right) \\
\quad \times \sin \left(\theta_{k}(0)+n t\right) d t .
\end{aligned}
$$

Obviously, we have the following:

$$
\begin{aligned}
& \int_{0}^{2 \pi} g\left(\frac{1}{n} r_{k}(0)^{1 / 2} \sin \left(\theta_{k}(0)+n(t-\tau)\right)+O(1)\right) \\
& \times \sin \left(\theta_{k}(0)+n t\right) d t \\
&= \frac{1}{n} \int_{\theta_{k}(0)-n \tau}^{2 n \pi+\theta_{k}(0)-n \tau} g\left(\frac{1}{n} r_{k}(0)^{1 / 2} \sin s+O(1)\right) \\
& \quad \times \sin (s+n \tau) d s \\
&=\frac{\cos n \tau}{n} \\
& \quad \times \int_{\theta_{k}(0)-n \tau}^{2 n \pi+\theta_{k}(0)-n \tau} g\left(\frac{1}{n} r_{k}(0)^{1 / 2} \sin s+O(1)\right) \sin s d s \\
& \quad+\frac{\sin n \tau}{n} \\
& \quad \times \int_{\theta_{k}(0)-n \tau}^{2 n \pi+\theta_{k}(0)-n \tau} g\left(\frac{1}{n} r_{k}(0)^{1 / 2} \sin s+O(1)\right) \cos s d s .
\end{aligned}
$$

Since $\theta_{k}(0) \in[0,2 \pi]$, there exists a subsequence $\left\{\theta_{k_{j}}(0)\right\}$ such that $\theta_{k_{j}} \rightarrow \theta_{*}, j \rightarrow \infty$. By using Lebesgue 
dominated convergent theorem and the condition $\left(h_{1}\right)$, we get the following:

$$
\begin{gathered}
\lim _{j \rightarrow+\infty} \int_{\theta_{k_{j}}(0)-n \tau}^{2 n \pi+\theta_{k_{j}}(0)-n \tau} g\left(\frac{1}{n} r_{k_{j}}(0)^{1 / 2} \sin s+O(1)\right) \sin s d s \\
=2 n(g(+\infty)-g(-\infty)), \\
\lim _{j \rightarrow+\infty} \int_{\theta_{k_{j}}(0)-n \tau}^{2 n \pi+\theta_{k_{j}}(0)-n \tau} g\left(\frac{1}{n} r_{k_{j}}(0)^{1 / 2} \sin s+O(1)\right) \cos s d s=0 .
\end{gathered}
$$

Therefore,

$$
\begin{gathered}
\lim _{j \rightarrow+\infty} \int_{0}^{2 \pi} g\left(x_{k_{j}}(t-\tau)\right) \sin \left(\theta_{k_{j}}(0)+n t\right) d t \\
=2 \cos n \tau[g(+\infty)-g(-\infty)] .
\end{gathered}
$$

Similarly, we can get the following:

$$
\lim _{j \rightarrow+\infty} \int_{0}^{2 \pi} F\left(x_{k_{j}}(t)\right) \cos \left(\theta_{k_{j}}(0)+n t\right) d t=0 .
$$

Hence, we obtain the following:

$$
2 \cos n \tau[g(+\infty)-g(-\infty)]=\int_{0}^{2 \pi} p(t) \sin \left(\theta_{*}+n t\right) d t
$$

which contradicts with (43).

On the other hand, it follows from (40) that

$$
\begin{aligned}
n \int_{0}^{2 \pi} & F\left(x_{k}(t)\right) \sin \left(\theta_{k}(0)+n t\right) d t \\
& \quad+\int_{0}^{2 \pi} g\left(x_{k}(t-\tau)\right) \cos \left(\theta_{k}(0)+n t\right) d t \\
& =\int_{0}^{2 \pi} p(t) \cos \left(\theta_{k}(0)+n t\right) d t .
\end{aligned}
$$

According to (50), we have the following:

$$
\begin{aligned}
\int_{0}^{2 \pi} g\left(x_{k}(t-\tau)\right) \cos \left(\theta_{k}(0)+n t\right) d t \\
=\int_{0}^{2 \pi} g\left(\frac{1}{n} r_{k}(0)^{1 / 2} \cos \left(\theta_{k}(0)+n(t-\tau)\right)+O(1)\right) \\
\quad \times \cos \left(\theta_{k}(0)+n t\right) d t \\
=\frac{1}{n} \int_{\theta_{k}(0)-n \tau}^{2 n \pi+\theta_{k}(0)-n \tau} g\left(\frac{1}{n} r_{k}(0)^{1 / 2} \sin s+O(1)\right) \\
=\frac{\cos n \tau}{n} \\
\quad \times \int_{\theta_{k}(0)-n \tau}^{2 n \pi+\theta_{k}(0)-n \tau} g\left(\frac{1}{n} r_{k}(0)^{1 / 2} \sin s+O(1)\right) \cos s d s \\
\quad-\frac{\sin n \tau}{n} \\
\quad \times \int_{\theta_{k}(0)-n \tau}^{2 n \pi+\theta_{k}(0)-n \tau} g\left(\frac{1}{n} r_{k}(0)^{1 / 2} \sin s+O(1)\right) \sin s d s
\end{aligned}
$$

From (53) we obtain the following:

$$
\begin{gathered}
\lim _{j \rightarrow+\infty} \int_{0}^{2 \pi} g\left(x_{k_{j}}(t-\tau)\right) \cos \left(\theta_{k_{j}}(0)+n t\right) d t \\
=-2 \sin n \tau[g(+\infty)-g(-\infty)] .
\end{gathered}
$$

Similarly, we have the following:

$$
\begin{aligned}
& \lim _{j \rightarrow+\infty} \int_{0}^{2 \pi} F\left(x_{k_{j}}(t)\right) \sin \left(\theta_{k_{j}}(0)+n t\right) d t \\
& \quad=2[F(+\infty)-F(-\infty)] .
\end{aligned}
$$

It follows from (57)-(60) that

$$
\begin{aligned}
2 n[F(+\infty)-F(-\infty)]-2 \sin (n \tau)[g(+\infty)-g(-\infty)] \\
\quad=\int_{0}^{2 \pi} p(t) \cos \left(\theta_{*}+n t\right) d t
\end{aligned}
$$

which contradicts with (44). Therefore, there exist positive constants $M_{1}$ and $M_{2}$ such that (45) holds.

(2) Let $x(t)=\varrho \sin (n t+\alpha)$, where $\alpha$ is an arbitrary constant. We will prove that there exists $M_{0}>0$ such that, for $\varrho \geq M_{0}, N x \notin \operatorname{Im} L$. Otherwise, there exits a sequence 
$\left\{\varrho_{k}\right\}$ satisfying $\lim _{k \rightarrow \infty} \varrho_{k}=+\infty$ such that $N x_{k} \in \operatorname{Im} L$ with $x_{k}(t)=\varrho_{k} \sin (n t+\alpha)$. We will prove the following:

$$
\begin{aligned}
& 2 \cos n \tau[g(+\infty)-g(-\infty)]=\int_{0}^{2 \pi} p(t) \sin (n t+\alpha) d t \\
& 2 n[F(+\infty)-F(-\infty)]-2 \sin (n \tau)[g(+\infty)-g(-\infty)] \\
& \quad=\int_{0}^{2 \pi} p(t) \sin (n t+\alpha) d t .
\end{aligned}
$$

In fact, since $N x_{k} \in \operatorname{Im} L$, we have the following:

$$
\begin{aligned}
& \int_{0}^{2 \pi}\left[f\left(x_{k}(t)\right) x_{k}^{\prime}(t)+g\left(x_{k}(t-\tau)\right)\right] \sin (n t+\alpha) d t \\
& \quad=\int_{0}^{2 \pi} p(t) \sin (n t+\alpha) d t \\
& \int_{0}^{2 \pi}\left[f\left(x_{k}(t)\right) x_{k}^{\prime}(t)+g\left(x_{k}(t-\tau)\right)\right] \cos (n t+\alpha) d t \\
& =\int_{0}^{2 \pi} p(t) \cos (n t+\alpha) d t .
\end{aligned}
$$

Using the same method as in step 1, we have the following:

$$
\begin{aligned}
& \lim _{k \rightarrow \infty} \int_{0}^{2 \pi}\left[f\left(x_{k}(t)\right) x_{k}^{\prime}(t)+g\left(x_{k}(t-\tau)\right)\right] \sin (n t+\alpha) d t \\
& \quad= 2 \cos n \tau[g(+\infty)-g(-\infty)] \\
& \lim _{k \rightarrow \infty} \int_{0}^{2 \pi}\left[f\left(x_{k}(t)\right) x_{k}^{\prime}(t)+g\left(x_{k}(t-\tau)\right)\right] \cos (n t+\alpha) d t \\
& \quad=2 n[F(+\infty)-F(-\infty)] \\
& \quad-2 \sin (n \tau)[g(+\infty)-g(-\infty)] .
\end{aligned}
$$

As a consequence, (62) holds. Thus, we get a contradiction.

(3) Let $M>\max \left\{n M_{0}, M_{1}, M_{2}\right\}$ be a sufficiently large constant (if it is necessary, $M$ can be enlarged). Set

$$
\Omega=\left\{x \in X:\|x\|_{\infty}<M,\left\|x^{\prime}\right\|_{\infty}<M\right\} .
$$

From the conclusion in step 1 we know that

$$
L x \neq \lambda N x, \quad \forall x \in \partial \Omega \cap D(L), \lambda \in(0,1) .
$$

From the conclusion in step 2 we know that

$$
N x \notin \operatorname{Im} L, \quad \forall x \in \partial \Omega \cap \operatorname{Ker} L,
$$

which implies $Q N x \neq 0$ for any $x \in \partial \Omega \cap \operatorname{Ker} L$. Since $\operatorname{Im} Q=$ Ker $L$, we can take an isomorphism $J=$ identity : $\operatorname{Im} Q \rightarrow$ Ker $L$. In what follows, we will prove the following:

$$
\operatorname{deg}\{J Q N, \Omega \cap \operatorname{Ker} L, 0\} \neq 0 \text {. }
$$

To this end, let us define $\phi: \operatorname{Ker} L \rightarrow \mathbf{R}^{2}, x=a \sin n t+$ $b \cos n t \rightarrow(a, b)$, namely,

$$
\phi x=(a, b) .
$$

Obviously, $\phi$ is a linear isomorphism. For any $x=a \sin n t+$ $b \cos n t$, set

$$
(J Q N x)(t)=h_{1}(a, b) \sin n t+h_{2}(a, b) \cos n t,
$$

where

$$
\begin{aligned}
& h_{1}(a, b)=\frac{1}{\pi} \int_{0}^{2 \pi} {\left[-f(x(t)) x^{\prime}(t)-g(x(t-\tau))+p(t)\right] } \\
& \times \sin n t d t \\
& h_{2}(a, b)=\frac{1}{\pi} \int_{0}^{2 \pi}\left[-f(x(t)) x^{\prime}(t)-g(x(t-\tau))+p(t)\right] \\
& \quad \times \cos n t d t .
\end{aligned}
$$

Define $h: \mathbf{R}^{2} \rightarrow \mathbf{R}^{2}$ as follows:

$$
h(a, b)=\phi \circ Q \circ N \circ \phi^{-1}(a, b)=\left(h_{1}(a, b), h_{2}(a, b)\right) .
$$

Then we have the following:

$\operatorname{deg}\{J Q N, \Omega \cap \operatorname{Ker} L, 0\}=\operatorname{deg}\{h, \phi(\Omega \cap \operatorname{Ker} L), 0\}$.

To calculate $\operatorname{deg}\{h, \phi(\Omega \cap \operatorname{Ker} L), 0\}$, we first estimate $l_{1}$ and $l_{2}$ as follows:

$$
\begin{aligned}
& l_{1}(a, b)=\frac{1}{\pi} \int_{0}^{2 \pi}\left[-f(x(t)) x^{\prime}(t)-g(x(t-\tau))\right] \sin n t d t, \\
& l_{2}(a, b)=\frac{1}{\pi} \int_{0}^{2 \pi}\left[-f(x(t)) x^{\prime}(t)-g(x(t-\tau))\right] \cos n t d t .
\end{aligned}
$$

Write $x=\rho \sin (n t+9)$ with $\rho=\sqrt{a^{2}+b^{2}}, \vartheta=\arctan (b / a)$ or $\vartheta=\pi+\arctan (b / a)$. Then we have that, for $\rho \rightarrow \infty$,

$$
\begin{aligned}
\int_{0}^{2 \pi} f(x(t)) x^{\prime}(t) \sin n t d t \\
=-n \int_{0}^{2 \pi} F(x(t)) \cos n t d t \\
=-n \int_{0}^{2 \pi} F(\rho \sin (n t+\vartheta)) \cos n t d t \\
=-\int_{\vartheta}^{2 n \pi+\vartheta} F(\rho \sin s)(\cos s \cos \vartheta+\sin s \sin \vartheta) d s \\
=-\cos \vartheta \int_{\vartheta}^{2 n \pi+\vartheta} F(\rho \sin s) \cos s d s \\
\quad-\sin \vartheta \int_{\vartheta}^{2 n \pi+\vartheta} F(\rho \sin s) \sin s d s \\
=-2 n \sin \vartheta[F(+\infty)-F(-\infty)]+o(1) .
\end{aligned}
$$


On the other hand, we have that, for $\rho \rightarrow \infty$,

$$
\begin{aligned}
& \int_{0}^{2 \pi} g(x(t-\tau)) \sin n t d t \\
&=\int_{0}^{2 \pi} g(\rho \sin (n(t-\tau)+\vartheta)) \sin n t d t \\
&=\frac{1}{n} \int_{\vartheta-n \tau}^{2 n \pi+9-n \tau} g(\rho \sin s) \sin (s+n \tau-\vartheta) d s \\
&=\frac{\cos (n \tau-\vartheta)}{n} \int_{\vartheta-n \tau}^{2 n \pi+\vartheta-n \tau} g(\rho \sin s) \sin s d s \\
& \quad+\frac{\sin (n \tau-\vartheta)}{n} \int_{\vartheta-n \tau}^{2 n \pi+\vartheta-n \tau} g(\rho \sin s) \cos s d s \\
&= 2 \cos (n \tau-\vartheta)[g(+\infty)-g(-\infty)]+o(1) .
\end{aligned}
$$

Therefore, we get the following:

$$
\begin{aligned}
l_{1}(a, b)=\frac{2}{\pi}\{n & \sin \vartheta[F(+\infty)-F(-\infty)] \\
& \quad-\cos (n \tau-\vartheta)[g(+\infty)-g(-\infty)]\} \\
& +o(1) .
\end{aligned}
$$

To estimate $l_{2}$, we have that, for $\rho \rightarrow \infty$,

$$
\begin{aligned}
& \int_{0}^{2 \pi} f(x(t)) x^{\prime}(t) \cos n t d t \\
&=n \int_{0}^{2 \pi} F(x(t)) \sin n t d t \\
&=n \int_{0}^{2 \pi} F(\rho \sin (n t+\vartheta)) \sin n t d t \\
&=\cos \vartheta \int_{\vartheta}^{2 n \pi+\vartheta} F(\rho \sin s) \sin s d s \\
&-\sin \vartheta \int_{\vartheta}^{2 n \pi+\vartheta} F(\rho \sin s) \cos s d s \\
&= 2 n \cos \vartheta[F(+\infty)-F(-\infty)]+o(1) .
\end{aligned}
$$

Meanwhile, we get that, for $\rho \rightarrow \infty$,

$$
\begin{aligned}
\int_{0}^{2 \pi} g & (x(t-\tau)) \cos n t d t \\
= & \int_{0}^{2 \pi} g(\rho \sin (n(t-\tau)+\vartheta)) \cos n t d t \\
= & \frac{1}{n} \int_{\vartheta-n \tau}^{2 n \pi+\vartheta-n \tau} g(\rho \sin s) \cos (s+n \tau-\vartheta) d s \\
= & \frac{\cos (n \tau-\vartheta)}{n} \int_{\vartheta-n \tau}^{2 n \pi+9-n \tau} g(\rho \sin s) \cos s d s \\
& -\frac{\sin (n \tau-\vartheta)}{n} \int_{\vartheta-n \tau}^{2 n \pi+9-n \tau} g(\rho \sin s) \sin s d s \\
= & -2 \sin (n \tau-\vartheta)[g(+\infty)-g(-\infty)]+o(1) .
\end{aligned}
$$

Hence, we obtain the following:

$$
\begin{aligned}
l_{2}(a, b)=\frac{2}{\pi}\{ & -n \cos \vartheta[F(+\infty)-F(-\infty)] \\
& +\sin (n \tau-\vartheta)[g(+\infty)-g(-\infty)]\} \\
& +o(1) .
\end{aligned}
$$

Set

$$
\begin{aligned}
& \widehat{h}_{1}(a, b)=\frac{2}{\pi}\{ n \sin \vartheta[F(+\infty)-F(-\infty)] \\
&\quad-\cos (n \tau-\vartheta)[g(+\infty)-g(-\infty)]\}, \\
& \widehat{h}_{2}(a, b)=\frac{2}{\pi}\{-n \cos \vartheta[F(+\infty)-F(-\infty)] \\
&+\sin (n \tau-\vartheta)[g(+\infty)-g(-\infty)]\}, \\
& \widehat{h}(a, b)=\left(\widehat{h}_{1}(a, b), \widehat{h}_{2}(a, b)\right) .
\end{aligned}
$$

Replacing $\vartheta$ in $x=\rho \sin (n t+\vartheta)$ with $\pi+\vartheta$, we get the following:

$$
\begin{aligned}
\widehat{h}_{1}(-a,-b)=-\frac{2}{\pi}\{ & n \sin \vartheta[F(+\infty)-F(-\infty)] \\
& -\cos (n \tau-\vartheta)[g(+\infty)-g(-\infty)]\}, \\
\widehat{h}_{2}(-a,-b)=-\frac{2}{\pi}\{ & -n \cos \vartheta[F(+\infty)-F(-\infty)] \\
& +\sin (n \tau-\vartheta)[g(+\infty)-g(-\infty)]\} .
\end{aligned}
$$

As a consequence,

$$
\begin{gathered}
\widehat{h}_{1}(-a,-b)=-\widehat{h}_{1}(a, b), \\
\widehat{h}_{2}(-a,-b)=-\widehat{h}_{2}(a, b), \\
\widehat{h}(-a,-b)=-\widehat{h}(a, b) .
\end{gathered}
$$

We note that, for $a^{2}+b^{2} \rightarrow+\infty$,

$$
\begin{aligned}
& h_{1}(a, b)=\widehat{h}_{1}(a, b)+c_{1}+o(1), \\
& h_{2}(a, b)=\widehat{h}_{2}(a, b)+c_{2}+o(1),
\end{aligned}
$$

where

$$
\begin{aligned}
& c_{1}=\frac{1}{\pi} \int_{0}^{2 \pi} p(t) \sin n t d t \\
& c_{2}=\frac{1}{\pi} \int_{0}^{2 \pi} p(t) \cos n t d t .
\end{aligned}
$$

Let us consider the map $H: \phi(\Omega \cap \operatorname{Ker} L) \times[0,1] \rightarrow \mathbf{R}^{2}:$ $(a, b, \mu) \rightarrow\left(h_{1}(a, b, \mu), h_{2}(a, b, \mu)\right)$ with

$$
\begin{aligned}
& h_{1}(a, b, \mu)=\widehat{h}_{1}(a, b)+\mu c_{1}, \\
& h_{2}(a, b, \mu)=\widehat{h}_{2}(a, b)+\mu c_{2} .
\end{aligned}
$$


Obviously, $H$ is continuous. Next, we shall prove that, for any $(a, b, \mu) \in \partial \phi(\Omega \cap \operatorname{Ker} L) \times[0,1]$,

$$
\left(h_{1}(a, b, \mu), h_{2}(a, b, \mu)\right) \neq(0,0) .
$$

Otherwise, there exists some $(a, b, \mu) \in \partial \phi(\Omega \cap \operatorname{Ker} L) \times[0,1]$ such that

$$
h_{1}(a, b, \mu)=0, \quad h_{2}(a, b, \mu)=0 .
$$

Then we have the following:

$$
\begin{aligned}
2 n \sin \vartheta & {[F(+\infty)-F(-\infty)] } \\
& -2 \cos (n \tau-\vartheta)[g(+\infty)-g(-\infty)] \\
= & -\mu \pi c_{1}, \\
-2 n \cos \vartheta[F(+\infty)-F(-\infty)] & \\
& +2 \sin (n \tau-\vartheta)[g(+\infty)-g(-\infty)] \\
= & -\mu \pi c_{2} .
\end{aligned}
$$

Therefore, we get the following:

$$
\begin{aligned}
& 2 \cos n \tau[g(+\infty)-g(-\infty)]=\mu \int_{0}^{2 \pi} p(t) \sin (n t+\vartheta) d t \\
& 2 n[F(+\infty)-F(-\infty)]-2 \sin (n \tau)[g(+\infty)-g(-\infty)] \\
& =\mu \int_{0}^{2 \pi} p(t) \cos (n t+\vartheta) d t .
\end{aligned}
$$

Since $\mu \in[0,1]$, we know from (90) that

$$
\begin{gathered}
2 \cos n \tau[g(+\infty)-g(-\infty)] \in[-\varrho, \varrho], \\
2 n[F(+\infty)-F(-\infty)] \\
-2 \sin (n \tau)[g(+\infty)-g(-\infty)] \in[-\varrho, \varrho],
\end{gathered}
$$

where $\varrho$ is given in Remark 2. From Remark 2 we know that (91) and (92) contradict with (43) and (44).

In particular, we have that, for $(a, b) \in \partial \phi(\Omega \cap \operatorname{Ker} L)$,

$$
\begin{gathered}
\left(\widehat{h}_{1}(a, b), \widehat{h}_{2}(a, b)\right) \neq(0,0), \\
\left(\widehat{h}_{1}(a, b)+c_{1}, \widehat{h}_{2}(a, b)+c_{2}\right) \neq(0,0) .
\end{gathered}
$$

Since $\widehat{h}: \mathbf{R}^{2} \rightarrow \mathbf{R}^{2}$ is odd and (93) holds, we know from Borsuk Theorem [12] that

$$
\operatorname{deg}(\widehat{h}, \phi(\Omega \cap \operatorname{Ker} L), 0)=2 m+1 \neq 0,
$$

where $m$ is an integer.

On the other hand, we know from (94) and the expressions of $\widehat{h}_{1}$ and $\widehat{h}_{2}$ that there exists a positive constant $\nu$, which is independent of $a$ and $b$, such that, for $(a, b) \in \mathbf{R}^{2}$

$$
\left|\widehat{h}_{1}(a, b)+c_{1}\right|+\left|\widehat{h}_{2}(a, b)+c_{2}\right| \geq v \text {. }
$$

Consequently, we infer from the homotopy invariance of degree that, if $M>\max \left\{M_{0}, M_{1}, M_{2}\right\}$ is large enough; then

$$
\begin{aligned}
\operatorname{deg} & (J Q N, \Omega \cap \operatorname{Ker} L, 0) \\
& =\operatorname{deg}(h, \phi(\Omega \cap \operatorname{Ker} L), 0) \\
& =\operatorname{deg}(H(\cdot, 1), \phi(\Omega \cap \operatorname{Ker} L), 0) \\
& =\operatorname{deg}(H(\cdot, 0), \phi(\Omega \cap \operatorname{Ker} L), 0) \\
& =\operatorname{deg}(\widehat{h}, \phi(\Omega \cap \operatorname{Ker} L), 0) \\
& =2 m+1 \neq 0 .
\end{aligned}
$$

Therefore, all conditions of Lemma 4 are satisfied. Thus, (1) has at least one $2 \pi$-periodic solution.

\section{Remarks}

We can use the method developed in Section 3 to deal with the existence of $2 \pi$-periodic solutions of the following equation:

$$
x^{\prime \prime}+f\left(x^{\prime}\right)+n^{2} x+g(x(t-\tau))=p(t) .
$$

Assume that the limits

$$
\left(h_{3}\right) \lim _{x \rightarrow \pm \infty} f(x)=f( \pm \infty)
$$

exist and are finite. We can prove the following theorem.

Theorem 5. Assume that the conditions $\left(h_{1}\right)$ and $\left(h_{3}\right)$ hold. Then (98) has at least one $2 \pi$-periodic solution provided that one of the following conditions holds:

$$
\begin{aligned}
& 2 \cos (n \tau)[g(+\infty)-g(-\infty)] \\
& \quad \neq \int_{0}^{2 \pi} p(t) \sin (\theta+n t) d t, \quad \forall \theta \in[0,2 \pi], \\
& 2 \cos (n \tau)[f(+\infty)-f(-\infty)] \\
& \quad \neq \int_{0}^{2 \pi} p(t) \sin (\theta+n t) d t, \quad \forall \theta \in[0,2 \pi], \\
& 2[g(+\infty)-g(-\infty)]-2 \sin (n \tau)[f(+\infty)-f(-\infty)] \\
& \quad \neq \int_{0}^{2 \pi} p(t) \sin (\theta+n t) d t, \quad \forall \theta \in[0,2 \pi], \\
& 2[f(+\infty)-f(-\infty)]-2 \sin (n \tau)[g(+\infty)-g(-\infty)] \\
& \quad \neq \int_{0}^{2 \pi} p(t) \sin (\theta+n t) d t, \quad \forall \theta \in[0,2 \pi] .
\end{aligned}
$$

Remark 6. In the case when $n=1$, the third and the fourth condition in Theorem 5 are identical to the related conditions in [6]. But the first and the second condition in Theorem 5 did not appear in [6]. 


\section{Acknowledgments}

The research was supported by Research Fund for the Doctoral Program of Higher Education of China, no. 11AA0013, Beijing Natural Science Foundation (Existence and multiplicity of periodic solutions in nonlinear oscillations), no. 1112006, and the Grant of Beijing Education Committee Key Project, no. KZ201310028031.

\section{References}

[1] X. Huang and Z. Xiang, "On the existence of $2 \pi$ periodic solutions of Duffing type equation $x^{\prime \prime}(t)+g(x(t-\tau))=p(t)$," Chinese Science Bulletin, vol. 39, pp. 201-203, 1994 (Chinese).

[2] W. G. Ge, "On the existence of harmonic solutions of Liénard systems," Nonlinear Analysis, vol. 16, no. 2, pp. 183-190, 1991.

[3] B. Liu and L. Huang, "Existence and uniqueness of periodic solutions for a kind of Liénard equation with a deviating argument," Applied Mathematics Letters, vol. 21, no. 1, pp. 5662, 2008.

[4] T. A. Burton and B. Zhang, "Boundedness, periodicity, and convergence of solutions in a retarded Liénard equation," Annali di Matematica Pura ed Applicata, vol. 165, pp. 351-368, 1993.

[5] S. Ma, Z. Wang, and J. Yu, "An abstract existence theorem at resonance and its applications," Journal of Differential Equations, vol. 145, no. 2, pp. 274-294, 1998.

[6] R. K. Nagle and M. E. Parrott, "Bounded perturbations with multiple delays of forced harmonic oscillators at resonance," Differential and Integral Equations, vol. 5, no. 6, pp. 1407-1418, 1992.

[7] S. Lu and W. Ge, "Periodic solutions for a kind of Liénard equation with a deviating argument," Journal of Mathematical Analysis and Applications, vol. 289, no. 1, pp. 231-243, 2004.

[8] B. Liu, "Boundedness in nonlinear oscillations at resonance," Journal of Differential Equations, vol. 153, no. 1, pp. 142-174, 1999.

[9] A. C. Lazer and D. E. Leach, "Bounded perturbations of forced harmonic oscillators at resonance," Annali di Matematica Pura ed Applicata, vol. 82, pp. 49-68, 1969.

[10] A. Capietto and Z. Wang, "Periodic solutions of Liénard equations at resonance," Differential and Integral Equations, vol. 16, no. 5, pp. 605-624, 2003.

[11] R. E. Gaines and J. L. Mawhin, Coincidence Degree, and Nonlinear Differential Equations, Springer, Berlin, Germany, 1977.

[12] D. Guo, Nonlinear Functional Analysis, Shandong science and technology press, 2002. 


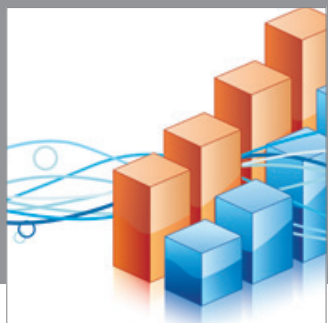

Advances in

Operations Research

mansans

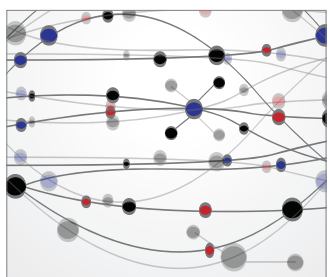

The Scientific World Journal
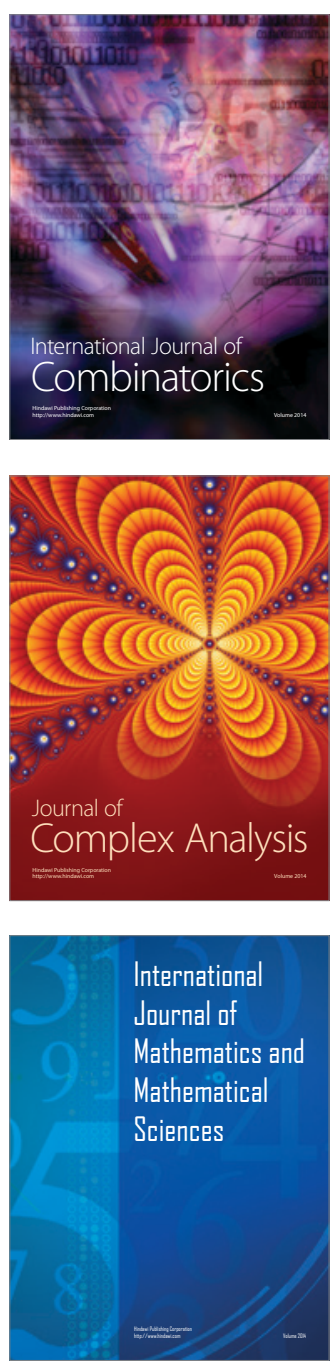
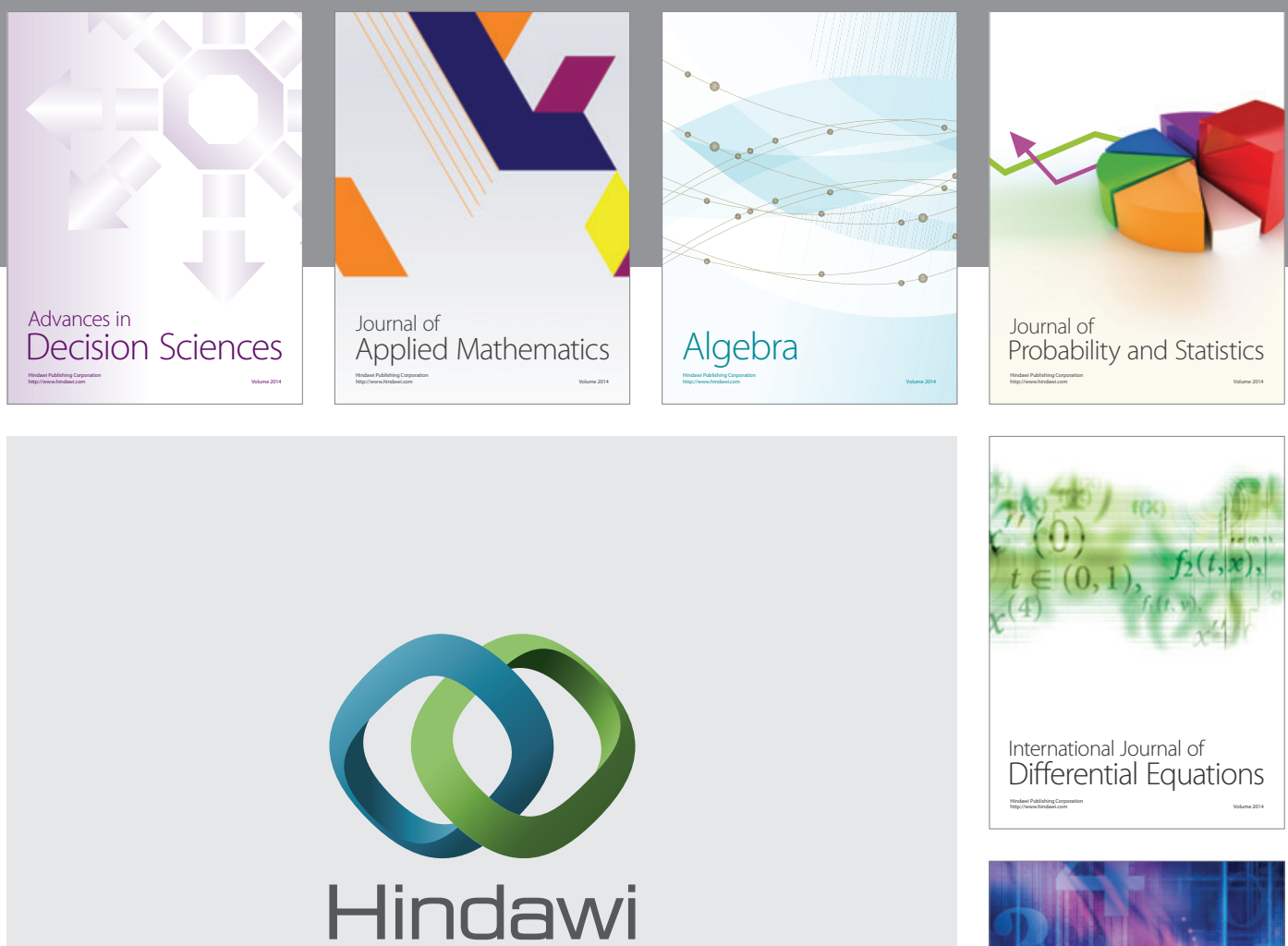

Submit your manuscripts at http://www.hindawi.com
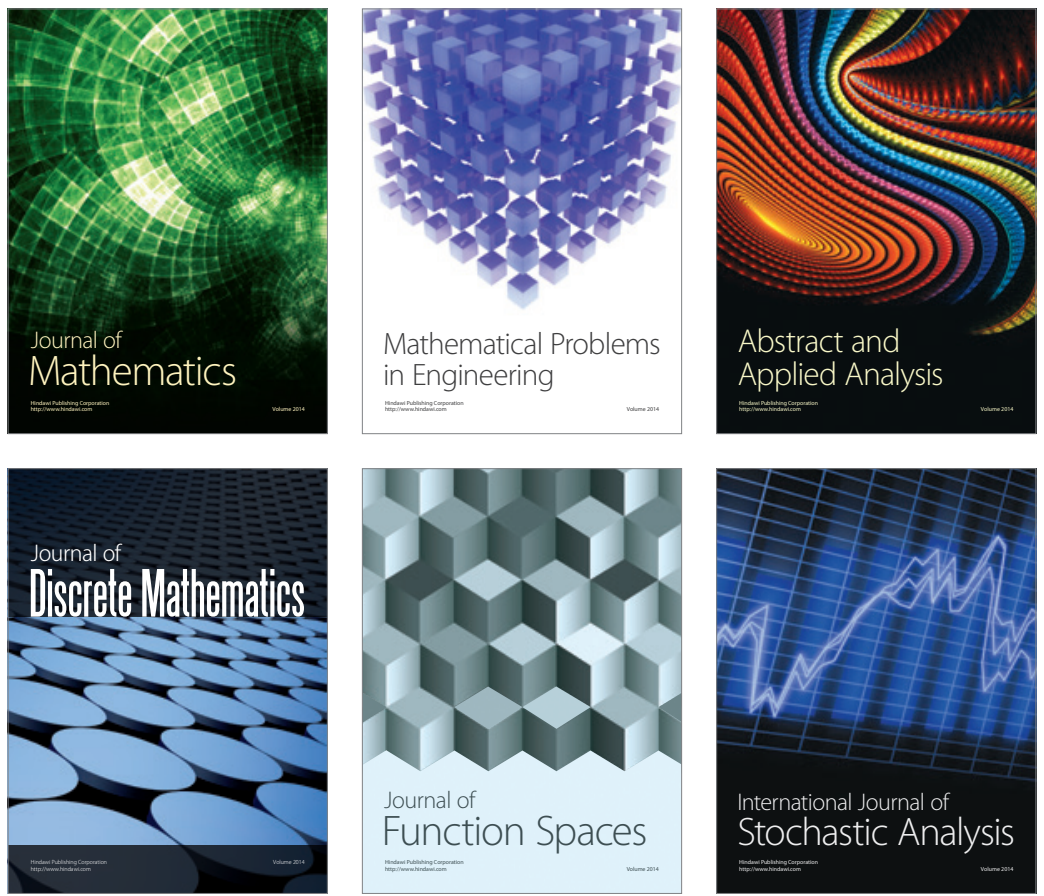

Journal of

Function Spaces

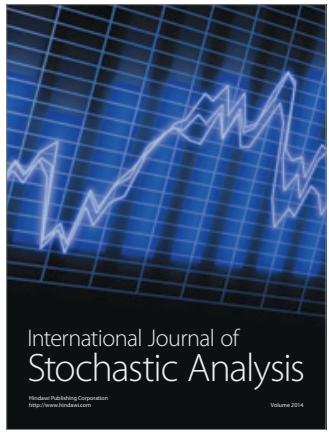

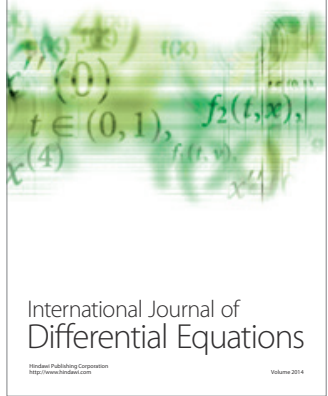
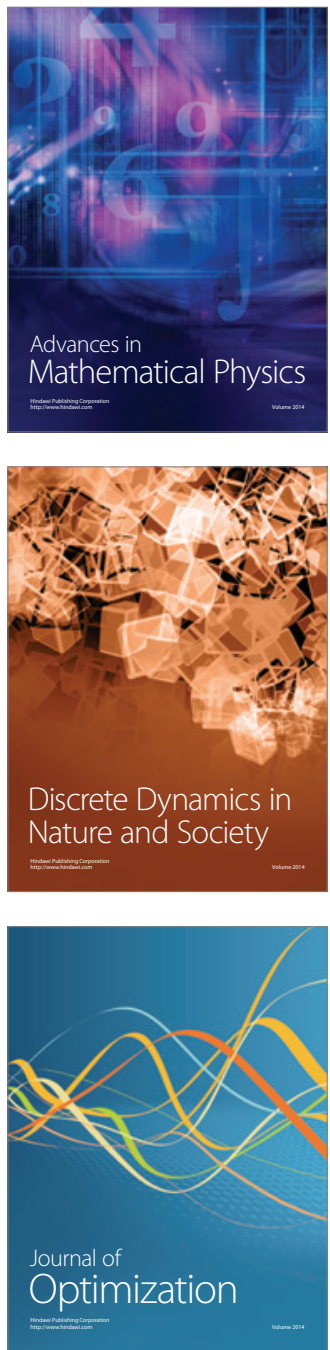